\title{
Identification of elasto-plastic characteristics by means of air-bending test
}

\author{
Luca Antonelli ${ }^{1}$, Pietro Salvini ${ }^{2}$, Francesco Vivio*, Vincenzo Vullo ${ }^{3}$ \\ Department of Mechanical Engineering, University of Rome “Tor Vergata”, Via del Politecnico 1, 00133 Rome, Italy \\ Received 4 April 2005; received in revised form 24 September 2006; accepted 29 September 2006
}

\begin{abstract}
A new identification method of material elasto-plastic characteristics by means of simple testing is proposed. The outcome of the procedure is the true-stress versus true-strain curve. The result is proposed in analytical form, according to Swift's law. The method requires acquisition of actual force-penetration values, carried out during a standard air-bending test. One of the main advantage of this method is that it allows an immediate identification of the material behaviour just in-process, that is to say that material characteristics can be accounted differently for each sheet subjected to bending; this is a fundamental preliminary step to achieve a bending adaptive control of sheets. Input requirements for identification include the geometry of tools and sheet, and the friction coefficient between sheet and die which needs to be estimated previously. In the proposed method, at a first stage the bending moment-curvature curve for the sheet is obtained by the numerical computation of force-penetration data through a multi-joint model of sheet during air-bending. Then, a simplified bending model, characterized by linear strain distribution across the thickness and plane-stress assumption, is employed to calculate the stress-strain curve.
\end{abstract}

(c) 2006 Elsevier B.V. All rights reserved.

Keywords: In-process material identification; Bending adaptive control; Springback compensation; Sheet bending model; Swift's law

\section{Introduction}

Correct evaluation of material behaviour is, like modelling accuracy and reliable representation of operative conditions, the most important aspect to consider in order to obtain suitable metal-forming analysis and simulations. Usually the evaluation of stress-strain curve is carried out by means of uni-axial tensile tests. By the knowledge of stress-strain curve or of the identified model parameters, it is possible to perform an elasto-plastic analysis simulating the process and providing useful information able to optimise, just in time, the technological process. Several air-bending models are already available in scientific literature, accounting of hardening behaviour in several forms, their complexity follows the approximation level required.

Some simplified models [1] use a bilinear hardening law considering the plastic behaviour governed by two parameters; an appreciable simplicity results in the analytical developments.

\footnotetext{
* Corresponding author. Tel.: +39 0672597123; fax: +39 062021351 .

E-mail addresses: antonelli@ing.uniroma2.it (L. Antonelli), salvini@uniroma2.it (P. Salvini), vivio@uniroma2.it (F. Vivio), vincenzo.vullo@uniroma2.it (V. Vullo).

1 Tel.: +390672597136.

2 Tel.: +390672597140.

3 Tel.: +390672597142 .
}

More accurately, other authors [2] consider the so called Ludwik's law $\left(\sigma=\sigma_{\mathrm{s}}+k \varepsilon^{z}\right)$; due to a three parameters description and its exponential nature, this law fits well the major part of metals paying a more complex solution process. However, the most widely diffuse hardening model is the Swift's one $\left(\sigma=\bar{C}\left(\varepsilon_{0}+\varepsilon_{\mathrm{p}}\right)^{\bar{e}}\right)$, employed in several semi-analytic or FE models [3-8].

Elasto-plastic material behaviour, as mentioned above, is usually carried out by uni-axial tensile test, but this kind of direct measurement is not always adequate; as a matter of facts, in some cases [9-12], it is better to turn on some kind of indirect test, mainly to point out particular aspects like Baushinger effects, dynamic influence and viscous properties.

The good agreement between experiments and simulations and, subsequently, the reliability of predictions are obviously, correlated to the correct and actual evaluation of material characteristics, so that, whenever possible, just in time material identification is desirable.

Generally, the identification of material characteristics for each workpiece is too expensive for industrial demand, being non-practical to perform uni-axial tensile tests of each one. In this context, any indirect in-process techniques able to detect the effective material is advantageous.

Confirming this last sentence, many proposed techniques are indirect, requiring the support of both analytical hypotheses and 


\begin{tabular}{|c|c|}
\hline \multicolumn{2}{|c|}{ Nomenclature } \\
\hline$C$ & $M-\chi$ hardening law coefficient \\
\hline $\bar{C}$ & Swift's $\sigma-\varepsilon$ law coefficient \\
\hline$d$ & punch penetration \\
\hline$e$ & $M-\chi$ hardening law exponent \\
\hline $\bar{e}$ & Swift's $\sigma-\varepsilon$ law exponent \\
\hline$E$ & Young's modulus \\
\hline$E \%$ & global error of the approximated deformation \\
\hline$F$ & punch load \\
\hline$k_{\mathrm{L}}$ & Ludwik's $\sigma-\varepsilon$ law coefficient \\
\hline$L$ & sheet depth \\
\hline$l$ & generic rigid element length between two joints \\
\hline $\bar{l}$ & semi-lengths sum of two consecutive elements \\
\hline$M$ & bending moment \\
\hline$n$ & number of rigid elements in the modelling \\
\hline$r$ & modelling length ratio $\left(l_{i} / l_{i-1}\right)$ \\
\hline$R_{d}$ & radius at sheet-die contact point \\
\hline$R_{m}$ & sheet middle radius \\
\hline$R_{n}$ & sheet neutral radius \\
\hline$t$ & sheet thickness \\
\hline$W$ & semi-die width \\
\hline$z$ & Ludwik's $\sigma-\varepsilon$ law exponent \\
\hline \multicolumn{2}{|c|}{ Greek symbols } \\
\hline$\chi$ & curvature \\
\hline$\varepsilon$ & strain \\
\hline$\varepsilon_{0}$ & deformation parameter in Swift's law \\
\hline$\varepsilon_{\mathrm{p}}$ & plastic strain \\
\hline$\mu$ & friction coefficient \\
\hline$\theta$ & element angle toward horizontal direction \\
\hline$\sigma_{\mathrm{s}}$ & material yield stress in Ludwik's law \\
\hline \multicolumn{2}{|c|}{ Subscripts } \\
\hline & element $i$ \\
\hline $\begin{array}{l}(i- \\
x, y, z\end{array}$ & $\begin{array}{l}i \text { elements } i-1 \text { and } i \\
\text { cartesian axises }\end{array}$ \\
\hline
\end{tabular}

calculations; however, they present the remarkable advantage to be available employing the same process set-up used for workpiece forming.

In particular, limiting these considerations on air-bending process, many methods [13-18] have been proposed in order to identify materials by means of simplified bending tests. Some of these $[13,15]$ have as main target the identification of material characteristics, others [14,16-18] make use of the identified results to optimise process efficiency, in terms of springback compensation and general process control.

The method proposed in this paper is an identification technique which, by means of a standard air-bending test, and in particular using force-penetration data measured during an airbending process of a sheet, calculates the numerical relationship between bending moment and curvature, and then, under planestress conditions, identifies the material elasto-plastic behaviour.
Future developments regard the connection between the identification phase and the prediction one, using the identification results to perform previsions on effective bending and springback compensation on a "sheet to sheet" basis. This goal is already reachable using the bending moment-curvature curve [18] but, more accurately, the purpose will be available using the stress-strain curve and discrete air-bending models derived by those proposed here.

This paper discusses modelling technique details, then the identification results carried out computing force-penetration curves generated through air-bending FE simulations, and finally, further identification results are examined taking input from an experimental air-bending test performed on a standard tensile test machine adequately equipped. Different material curves are employed for FE simulations and compared with the identified ones.

The method presents the advantage to be appropriate for the industrial process of sheet air-bending, in terms of springback compensation that, adopting this technique, would take advantage by material identification on an each-sheet basis. This would be particularly significant if bimetallic sheets were accounted, or if sheets were subjected to significant surface coating or treatments. In other words, the determination of the sheet behaviour in terms of bending moment-curvature is always possible even if non-homogeneity occurs. In these last cases, the resulting stress-strain curve should be adequately considered; as a matter of fact it is only a representative balanced average of the various constituent material.

Some FEM validation is presented in terms of comparison between identified and target entity, and finally an experimental application is illustrated.

\section{Theoretical approach}

\subsection{Multi-joint model}

In order to reduce mathematical complexity, some assumptions are accounted in the model approach; they regard sheet-die friction, sheet elongation and contact indentation.

Friction is accounted by means of a constant coefficient, according to Coulomb hypothesis; the value is not tuned during the identification process, so that a suitable method to determinate this coefficient should be previously employed. However, a modelling extension which accounts the identification of friction coefficient is theoretically possible increasing the number of in-process input data, but at the present stage of the research the technique appears too difficult to implement in real time. Middle plane sheet elongation and contact indentation are neglected because they weakly affect the results, as some refined finite element analysis suitably performed, showed clearly.

The envisaged model is characterized by localized deformability concentrated on elasto-plastic joints, connected each other by rigid elements (Fig. 1). These rigid portions are quantitatively and dimensionally defined by the $n$ elements covering the distance between punch and die, and their respective length ratio $r$ (Fig. 1a). The length ratio $r$ defines the geometric progression of element length from punch to die. This feature accords per- 

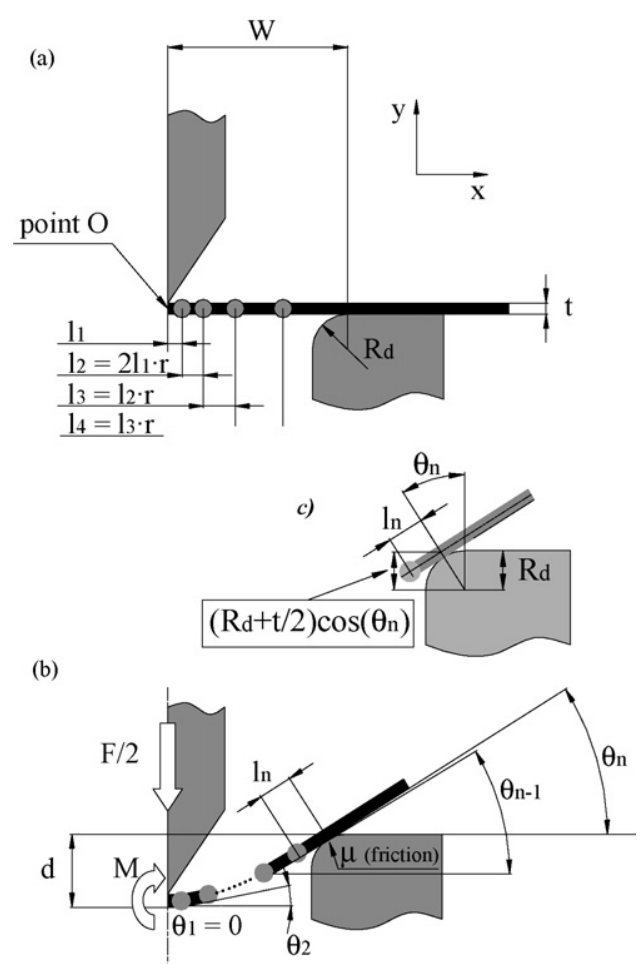

Fig. 1. Multi-joint model of the sheet during the bending: (a) before loading phase; (b) during loading phase; (c) loading phase zoom.

fectly with the necessity to increase discretization in the region where the curvature changes more quickly, the punch nose zone, where an higher element concentration provides a better accord between model and experimental displacement. Although the infinite number of degree of freedom of the sheet under bending is not reachable, great number of elements (20-50) and adequate values for $r$ (2-4), supply satisfactory results [18].

Elements thickness is taken from the real sheet, but in the model it has only a cinematic valence, since the deformation is accounted only in the elasto-plastic joints.

Since the identification procedure starts determining the joints unknown elasto-plastic behaviour using data coming from the working press-brake, it is important to formulate a generic mathematic law, with unknown parameters, able to adapt to the material behaviour in terms of bending moment-curvature. The chosen law is a simplification of most common hardening laws (Ludwik, Swift), usually providing stress-strain relations. The law is shown and elaborated to obtain the discretized form in Eq. (1).

$M=C \cdot \chi^{e}=C \cdot\left[\frac{\mathrm{d} \theta}{\mathrm{d} l}\right]^{e}$ or, in the discretized form,

$M_{(i-1)-i}=C \cdot\left[\chi_{(i-1)-i}\right]^{e}=C \cdot\left[\frac{\theta_{(i-1)-i}}{\bar{l}_{i-1}}\right]^{e}$

where

$\bar{l}_{i-1}=\frac{l_{i-1}}{2}+\frac{l_{i}}{2}$

The relation (1) is advantageous because it describes adequately the sheet behaviour managing only two unknown variables: $C$ and $e$. This is a very important aspect in consideration of the hard arithmetical manipulations and the non-linear numeric solutions that the whole procedure presents.

As usual in physical problems, the governing equations consider geometry conditions, equilibrium equations and constitutive relationship.

First geometric condition is in vertical direction and regards punch penetration (Fig. 1b and c):

$$
\begin{aligned}
& l_{1} \sin \left(\theta_{1}\right)+l_{2} \sin \left(\theta_{2}\right)+\cdots+l_{n} \sin \left(\theta_{n}\right)+\frac{t}{2} \\
& \quad+R_{d}-\left(R_{d}+\frac{t}{2}\right) \cos \left(\theta_{n}\right)=d
\end{aligned}
$$

Then on horizontal direction, considering semi-die width (Fig. $1 \mathrm{~b}$ and c):

$$
\begin{aligned}
& l_{1} \cos \left(\theta_{1}\right)+l_{2} \cos \left(\theta_{2}\right)+\cdots+l_{n} \cos \left(\theta_{n}\right) \\
& +\left(R_{d}+\frac{t}{2}\right) \sin \left(\theta_{n}\right)=W
\end{aligned}
$$

Other geometric conditions link the element angular positions to their proper rotations:

$\theta_{1}=0$

$\theta_{2}=\theta_{1}+\theta_{1-2}$

$\theta_{n}=\theta_{n-1}+\theta_{(n-1)-n}$

It should be highlighted that the first equation in (4) $\left(\theta_{l}=0\right)$ allows to avoid edged points below the punch, like the real evidence requires. This imposition does not affect the correct modelling shape during the bending because the first element has usually a very limited length.

At the intersection between the sheet horizontal axis and the punch vertical one (point $\mathrm{O}$ in Fig. 1a) it is possible to write the first rotation equilibrium equation (5) with the hypothesis that all the forces are not distributed but concentrated. This equation is written considering the sheet-die contact forces, accounting the orthogonal one and the friction one (tangent), and their vertical equilibrium with the punch force $F$.

$$
F=\frac{2 M}{\left(( ( \operatorname { t a n } ( \theta _ { n } ) - \mu ) / ( 1 + \mu \operatorname { t a n } ( \theta _ { n } ) ) ) \left(d-(t / 2)-R_{d}\right.\right.}
$$

Other rotation equilibrium equations are available at the joints connecting the rigid elements. The notation $M_{(i-1)-i}$ is used to indicate in Eq. (6) the bending moment at the joint between two elements:

$$
\begin{aligned}
& M_{1-2}=M-\frac{F}{2} \cdot l_{1} \cos \left(\theta_{1}\right) \\
& M_{2-3}=M-\frac{F}{2} \cdot\left[l_{1} \cos \left(\theta_{1}\right)+l_{2} \cos \left(\theta_{2}\right)\right] \\
& \vdots \\
& M_{(n-1)-n}=M-\frac{F}{2} \cdot\left[l_{1} \cos \left(\theta_{1}\right)+l_{2} \cos \left(\theta_{2}\right)+\cdots+\right. \\
& \left.\quad l_{n-1} \cos \left(\theta_{n-1}\right)\right]
\end{aligned}
$$


Finally, giving to $\bar{l}_{i}$ the same meaning given in Eq. (1), it is possible to write the constitutive relationship between angular variation and bending moment at all joints using the general applied hardening law (1).

$\theta_{1-2}=\bar{l}_{1}\left(\frac{1}{C} M_{1-2}\right)^{1 / e}$
$\theta_{2-3}=\bar{l}_{2}\left(\frac{1}{C} M_{2-3}\right)^{1 / e}$

$\theta_{(n-1)-n}=\bar{l}_{n-1}\left(\frac{1}{C} M_{(n-1)-n}\right)^{1 / e}$

Input data coming from the actual air-bending process are force and corresponding penetration $(F-d)$; calling $m$ the number of experienced $F-d$ values it is possible to count the number of available equations:

\begin{tabular}{lll}
\hline Punch penetration & $(2)$ & $1 \cdot m$ \\
Semi-die width & $(3)$ & $1 \cdot m$ \\
Angular positions & $(4)$ & $n \cdot m$ \\
Punch nose rotation eq. & $(5)$ & $1 \cdot m$ \\
Joints rotation equilibrium & $(6)$ & $(n-1) \cdot m$ \\
Applied hardening law & $(7)$ & $(n-1) \cdot m$ \\
\hline
\end{tabular}

With the same hypothesis this is the situation for unknown variables:

\begin{tabular}{ll}
\hline (a) $\theta_{i}$ & $n \cdot m$ \\
(b) $M$ & $1 \cdot m$ \\
(c) $l_{n}$ & $1 \cdot m$ \\
(d) $M_{(i-1)-i}$ & $(n-1) \cdot m$ \\
(e) $\theta_{(i-1)-i}$ & $(n-1) \cdot m$ \\
(f) $C, e$ & 2
\end{tabular}

Concluding, the problem presents the following number of equations and variables: equations: $3 n \cdot m+m$

unknown variables: $3 n \cdot m+2$

The solution is theoretically possible if $m=2$; in other words, two measurements are required to obtain as much as necessary information to perform the identification; in general, the minimum measurements number should be equal to the number of parameters describing the bending moment-curvature hardening law ( 2 in this case, $C$ and $e$ ). Practically, the problem is overdetermined and the number of measurements $m$ is bigger than 2 . The adopted strategy requires to solve the system of equation several times on couples of force-penetration data, then all results are composed to get an unique analytical hardening law. It should be pointed out, however, that it is possible in principle to get an approximated solution accounting for only two measurements; this highly error-affected way of operating is much quicker and could be useful for in-process identification.

Within the elastic behaviour region one should impose $e=1$ in the same law (1), this involves the requirement of only 1 couple of data $F-d$, i.e. $m=1$.

Eqs. (2)-(7) can be handled with the aim to reduce the number of unknown variables. As a matter of fact, in the equations group (4) disappears the already discussed condition $\theta_{1}=0$ and consequently the variable $\theta_{1}$ is substituted by 0 in all equations. Maximum bending moment $M$ appearing in Eqs. (6) can be calculated handing the Eqs. (5) and the resulting Eqs. (6) can be put in the hardening relations (7) in terms of bending moments at joints $M_{(i-1)-i}$. Analysing now Eqs. (4) it is possible to substitute the angular increments $\theta_{(i-1)-i}$ using those that relations (7) provide after the introduction of the $M_{(i-1)-i}$ expressions. Finally the length of the last element $l_{n}$, which appearing in (2), can be extracted by manipulations from Eq. (3), reducing again the number of unknown variables and equations.

As a result of all these manipulations, taking, for the seek of simplicity the number $n$ of rigid portions equal to 4 (usually $n=20-50$ ), considering $m=2$, and taking into account that symbol (1/2) indicates the function evaluated at the first/second state $(F(1 / 2)-d(1 / 2))$, the full mathematical problem appears as:

$$
\begin{aligned}
& \left\{\begin{array}{l}
l_{2} \sin \theta_{2}(1)+l_{3} \sin \theta_{3}(1)+\frac{t}{2}+R_{d}-\left(R_{d}+\frac{t}{2}\right) \cos \theta_{4}(1)+\left[W-\left(R_{d}+\frac{t}{2}\right) \sin \theta_{4}(1)-l_{1}-l_{2} \cos \theta_{2}(1)-l_{3} \cos \theta_{3}(1)\right] \tan \theta_{4}(1)=d(1) \\
l_{2} \sin \theta_{2}(2)+l_{3} \sin \theta_{3}(2)+\frac{t}{2}+R_{d}-\left(R_{d}+\frac{t}{2}\right) \cos \theta_{4}(2)+\left[W-\left(R_{d}+\frac{t}{2}\right) \sin \theta_{4}(2)-l_{1}-l_{2} \cos \theta_{2}(2)-l_{3} \cos \theta_{3}(2)\right] \tan \theta_{4}(2)=d(2)
\end{array}\right. \\
& \theta_{2}(1)=\bar{l}_{1}\left\{\frac{F(1)}{2 C}\left[\frac{\tan \theta_{4}(1)-\mu}{1+\mu \tan \theta_{4}(1)}\left(d(1)-\frac{t}{2}-R_{d}\left(1-\cos \theta_{4}(1)\right)\right)+W-R_{d} \sin \theta_{4}(1)-l_{1}\right]\right\}^{1 / e} \\
& \theta_{2}(2)=\bar{l}_{1}\left\{\frac{F(2)}{2 C}\left[\frac{\tan \theta_{4}(2)-\mu}{1+\mu \tan \theta_{4}(2)}\left(d(2)-\frac{t}{2}-R_{d}\left(1-\cos \theta_{4}(2)\right)\right)+W-R_{d} \sin \theta_{4}(2)-l_{1}\right]\right\}^{1 / e} \\
& \theta_{3}(1)=\theta_{2}(1)+\bar{l}_{2}\left\{\frac{F(1)}{2 C}\left[\frac{\tan \theta_{4}(1)-\mu}{1+\mu \tan \theta_{4}(1)}\left(d(1)-\frac{t}{2}-R_{d}\left(1-\cos \theta_{4}(1)\right)\right)+W-R_{d} \sin \theta_{4}(1)-\left(l_{1}+l_{2} \cos \theta_{2}(1)\right)\right]\right\}^{1 / e} \\
& \theta_{3}(2)=\theta_{2}(2)+\bar{l}_{2}\left\{\frac{F(2)}{2 C}\left[\frac{\tan \theta_{4}(2)-\mu}{1+\mu \tan \theta_{4}(2)}\left(d(2)-\frac{t}{2}-R_{d}\left(1-\cos \theta_{4}(2)\right)\right)+W-R_{d} \sin \theta_{4}(2)-\left(l_{1}+l_{2} \cos \theta_{2}(2)\right)\right]\right\}^{1 / e} \\
& \theta_{4}(1)=\theta_{3}(1)+\bar{l}_{3}\left\{\frac{F(1)}{2 C}\left[\frac{\tan \theta_{4}(1)-\mu}{1+\mu \tan \theta_{4}(1)}\left(d(1)-\frac{t}{2}-R_{d}\left(1-\cos \theta_{4}(1)\right)\right)+W-R_{d} \sin \theta_{4}(1)-\left(l_{1}+l_{2} \cos \theta_{2}(1)+l_{3} \cos \theta_{3}(1)\right)\right]\right\}^{1 / e} \\
& \theta_{4}(2)=\theta_{3}(2)+\bar{l}_{3}\left\{\frac{F(2)}{2 C}\left[\frac{\tan \theta_{4}(2)-\mu}{1+\mu \tan \theta_{4}(2)}\left(d(2)-\frac{t}{2}-R_{d}\left(1-\cos \theta_{4}(2)\right)\right)+W-R_{d} \sin \theta_{4}(2)-\left(l_{1}+l_{2} \cos \theta_{2}(2)+l_{3} \cos \theta_{3}(2)\right)\right]\right\}^{1 / e}
\end{aligned}
$$




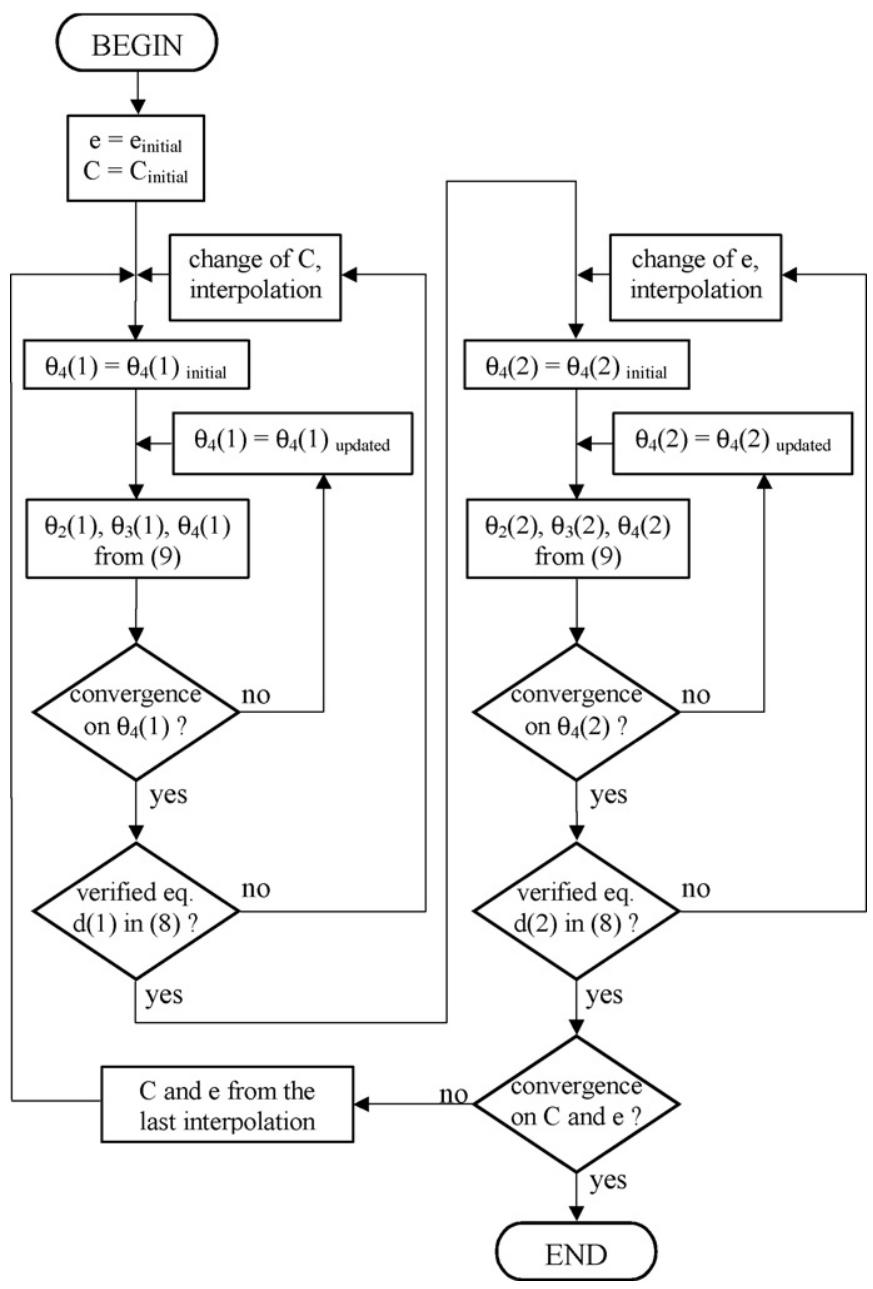

Fig. 2. Method for numeric solution.

The eight equations (8) and (9) present eight unknown variables: three angular positions at the state $1\left(\theta_{1}(1), \theta_{2}(1), \theta_{3}(1)\right)$, three angular positions at the state $2\left(\theta_{1}(2), \theta_{2}(2), \theta_{3}(2)\right)$, and parameters $C$ and $e$, which are invariable for the two states considered. The analytical solution of this non-linear system is very hard, but it is possible to get a converged solution using various numeric iterations organized on three levels, considering sequentially the two couples of measurements $F(1)-d(1), F(2)-d(2)$. The method is shown in Fig. 2 by means of a flow diagram. The diagram shows the sequential use of the two couples of measurements $F(1)-d(1), F(2)-d(2)$; first the initial values of $C$ and $e$ are imposed, then two similar phases are used to obtain the convergence on $C$ first and on $e$ consequently. In these phases the convergence is obtained when first (second) Eq. (8) is verified, and the iteration values of $C(e)$ are calculated by interpolation. The value of $C(e)$ is calculated considering as input variables at each step the values $e(C)$ calculated by the previous phase; this involves that the initial error deriving from the choice of $C_{\text {initial }}$ and $e_{\text {initial }}$ requires some iterations to vanish. As a matter of fact, the procedure does not stop until two consecutive calculations of $C$ and $e$ present approximately the same results.

It should be highlighted that the two described phases need the calculation of all the angles $\theta_{i}(i=1.4)$, and this is possi- (a)
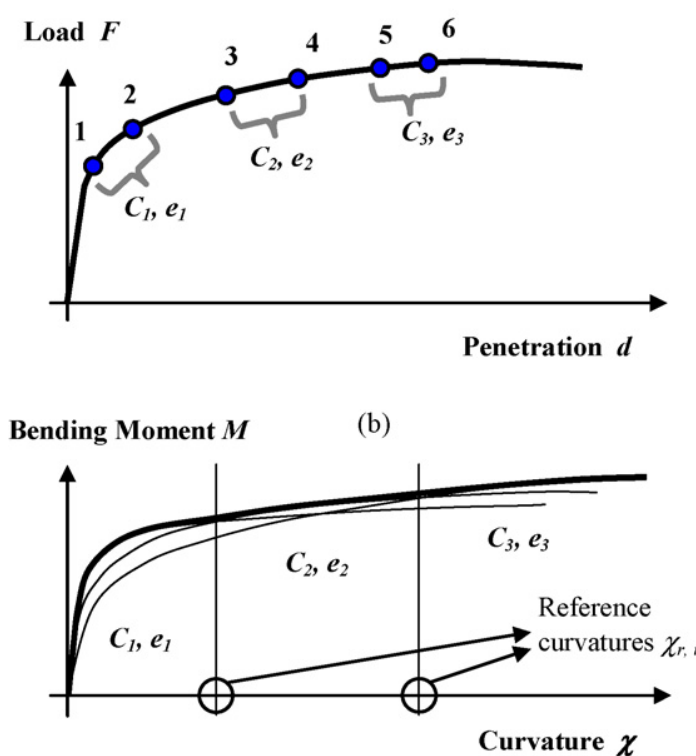

Fig. 3. Moment-curvature law composition: (d) force-penetration input curve; (e) composition of moment-curvature curve portions.

ble only by means of an other iterative calculation based on the choice of an initial value of $\theta_{4}$ and the consequent computation of all angles $\left(\theta_{4}\right.$ included). The iteration continues until the convergence on $\theta_{4}$ is reached. The method does not change conceptually when 20-50 elements are used instead the 4 introduced in this example; obviously, the identification becomes harder and more time is consumed.

Relations (8) and (9) are written for two couples of measurements and give the problem solution in term of parameters $C$ and $e$ describing the bending moment-curvature law corresponding analytically to the two measurements taken. Obviously, it is necessary to consider more than two measurements, and the best experimented method to obtain a good agreement between identified results and FEM data is to repeat the determination of $C$ and $e$ using different zones of the force-penetration curve coming from the process. Operating in this way, a family of curves can be obtained, and consequently it is possible to joint the relative curve portions extracted from the found $M-\chi$ curves (Fig. 3b). In other words the resulting bending moment-curvature curve is the composition of different curve portions, each one carried out elaborating a couple of measured point on the experimental (FEM in this case) force-penetration curve (Fig. 3a). Operating in this way it is necessary to define the validity fields for each

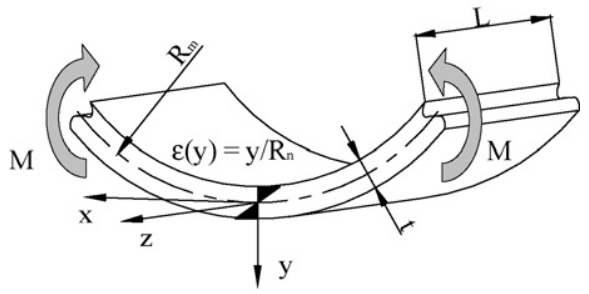

Fig. 4. Analytical bending model. 


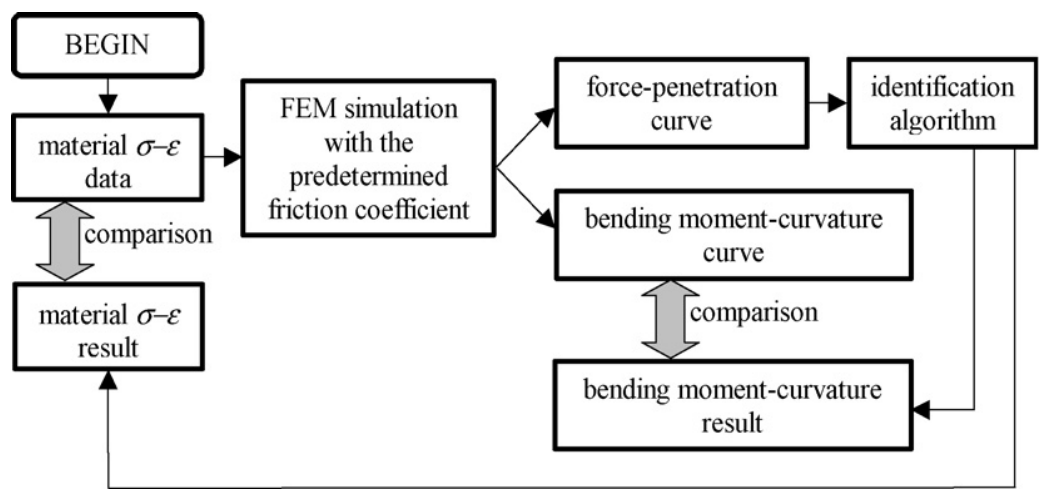

Fig. 5. Comparison with FEM analysis.
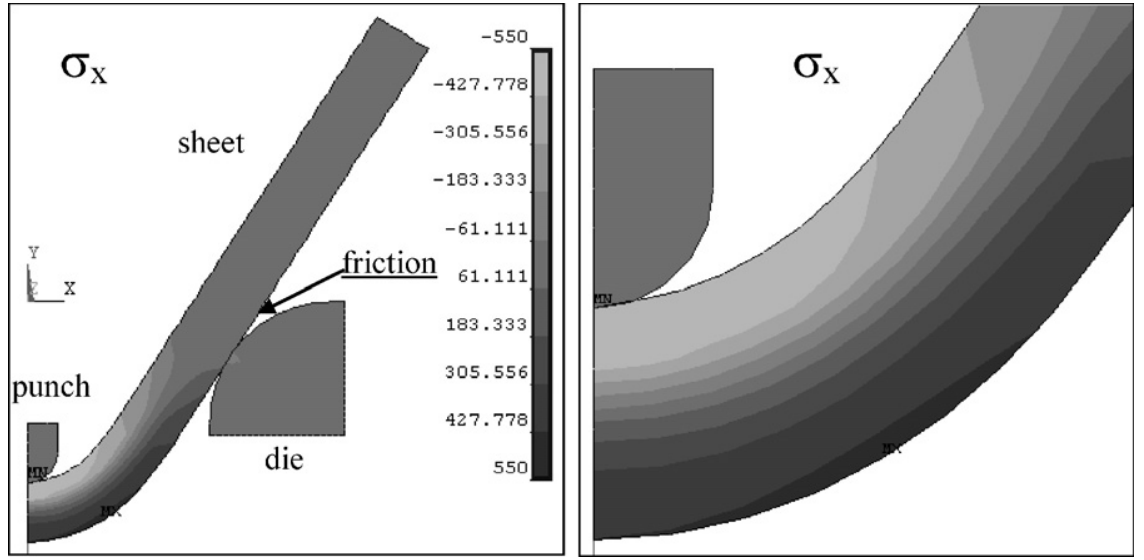

Fig. 6. FEM simulation at maximum punch penetration.

curve of the obtained family curve. These fields are determined by means of a reference curvature (Fig. 3b). The mentioned reference curvatures are those experienced by point $\mathrm{O}$ (corresponding to the maximum value) when the punch position lies between points $2 i$ and $2 i+1(i=1 \ldots(m / 2)-1)($ Fig. 3a). This

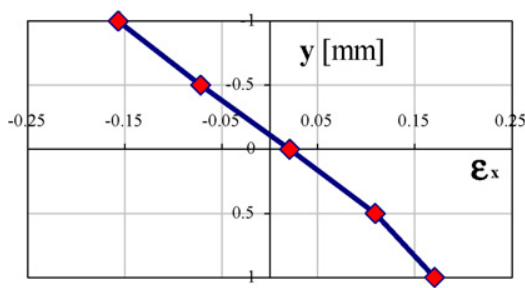

(a): $\boldsymbol{X}=0.210314 \mathrm{~mm}^{-1}$

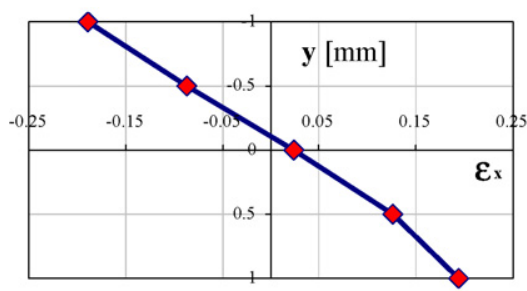

(b): $\boldsymbol{\chi}=0.243910 \mathrm{~mm}^{-1}$ technique allows to overcome the relative simplicity of the hardening law (1) since the identification, although obtained by a simplified law, is repeated several times for different bending levels, and the consequent results composition can fit greatly the FEM bending moment-curvature curve.

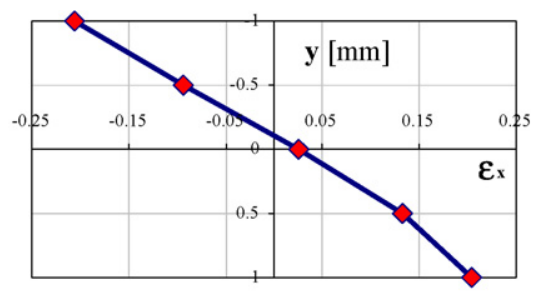

(c): $\boldsymbol{X}=0.259831 \mathrm{~mm}^{-1}$

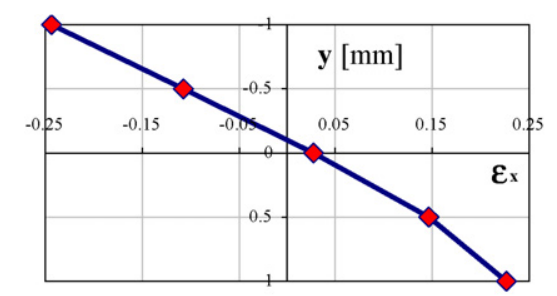

(d): $\boldsymbol{X}=0.291053 \mathrm{~mm}^{-1}$

Fig. 7. Deformation across the thickness for increasing curvatures. 


\subsection{Bending model}

When bending moment-curvature identification is performed and the various $M-\chi$ laws are locally calculated by the method discussed above, it is necessary to formulate a bending model which allows stress-strain identification.

Main assumptions are: linear deformation across the thickness, plane-stress mode. The first hypothesis involves that neutral radius $R_{n}$ (Fig. 4) coincides with the middle one $R_{m}$ and this, as will be shown later, is compatible only with simplified material laws (10), similar to law (1):

$\sigma=\bar{C} \cdot \varepsilon^{\bar{e}}$

Further consequence of the first assumption is the limitation of the identification results within relatively small deformation, in particular, calculating the error $E \%$ by respect to logarithmic deformation $\varepsilon_{\log }$ obtained using the approximation $R_{n} \approx R_{m}$ :

$$
\begin{aligned}
& \varepsilon_{\log }=\ln \left(1+\frac{y}{R_{n}}\right) \approx \ln \left(1+\frac{y}{R_{m}}\right)=\ln (1+\varepsilon) \\
& E \%=\frac{\varepsilon-\varepsilon_{\log }}{\varepsilon_{\log }} \times 100 \approx \frac{\varepsilon-\ln (1+\varepsilon)}{\ln (1+\varepsilon)} \times 100
\end{aligned}
$$

\section{Input data}

$$
\begin{aligned}
& E\left[N / m^{2}\right]=205.810^{9} \\
& \sigma\left[N / m^{2}\right]=720.8510^{6}\left(0.06+\varepsilon_{p}\right)^{0.3282}
\end{aligned}
$$

\section{Identified results}

$$
\begin{aligned}
& E\left[\mathrm{~N} / \mathrm{m}^{2}\right]=205.210^{9} \\
& \sigma\left[\mathrm{N} / \mathrm{m}^{2}\right]=70210^{6}\left(0.057+\varepsilon_{p}\right)^{0.3117}
\end{aligned}
$$

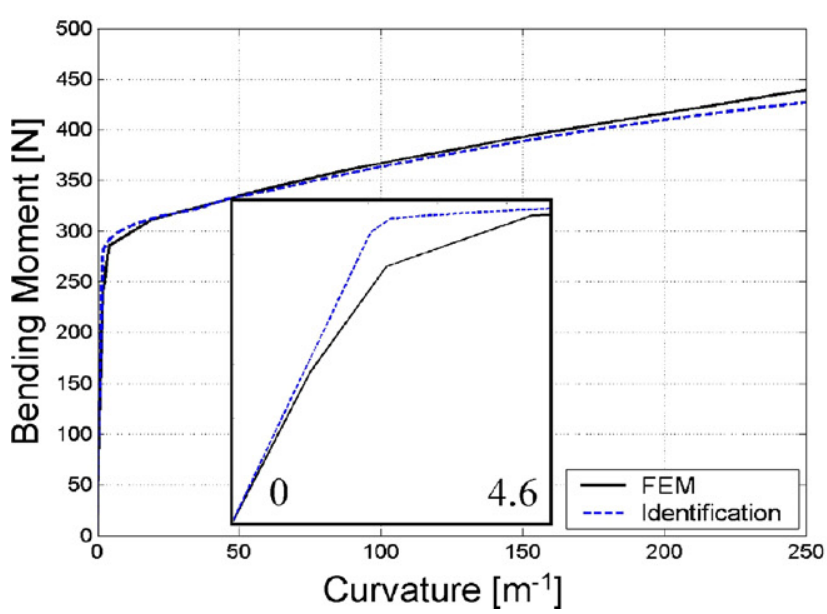

the identification results are taken within values of $E \%=12 \%$, i.e. $\varepsilon=0.25$.

Second assumption limits the test to the sheets where the dimension $L$ (Fig. 4), is not too much larger than the thickness $t$, so that $\sigma_{z}$ stress can be neglected.

If this second condition is not respected, the identified stress-strain behaviour is simply the one checked along $x$ direction (Fig. 4) under plane strain condition. In other word, the method is however applicable, but the results cannot be considered valid for material stress-strain identification.

With this assumptions it is possible to calculate the bending moment versus curvature (for unit length) on the sheet and to link the $\sigma-\varepsilon$ parameters in Eq. (10), with the $M-\chi$ ones in (1):

$$
\begin{aligned}
M & =2 \int_{0}^{t / 2} \sigma \cdot y \cdot \mathrm{d} y=2 \int_{0}^{t / 2} \bar{C} \cdot \varepsilon^{\bar{e}} \cdot y \cdot \mathrm{d} y \\
& =2 \cdot \bar{C} \cdot \int_{0}^{t / 2}\left(\frac{y}{R_{n}}\right)^{\bar{e}} \cdot y \cdot \mathrm{d} y=\frac{2 \cdot \bar{C}}{R_{n}^{\bar{e}}} \cdot \int_{0}^{t / 2} y^{\bar{e}+1} \cdot \mathrm{d} y \\
& =2 \cdot \bar{C} \cdot \chi^{\bar{e}} \cdot \int_{0}^{t / 2} y^{\bar{e}+1} \cdot \mathrm{d} y=\frac{2 \cdot \bar{C}}{\bar{e}+2} \cdot\left(\frac{t}{2}\right)^{\bar{e}+2} \cdot \chi^{\bar{e}}
\end{aligned}
$$
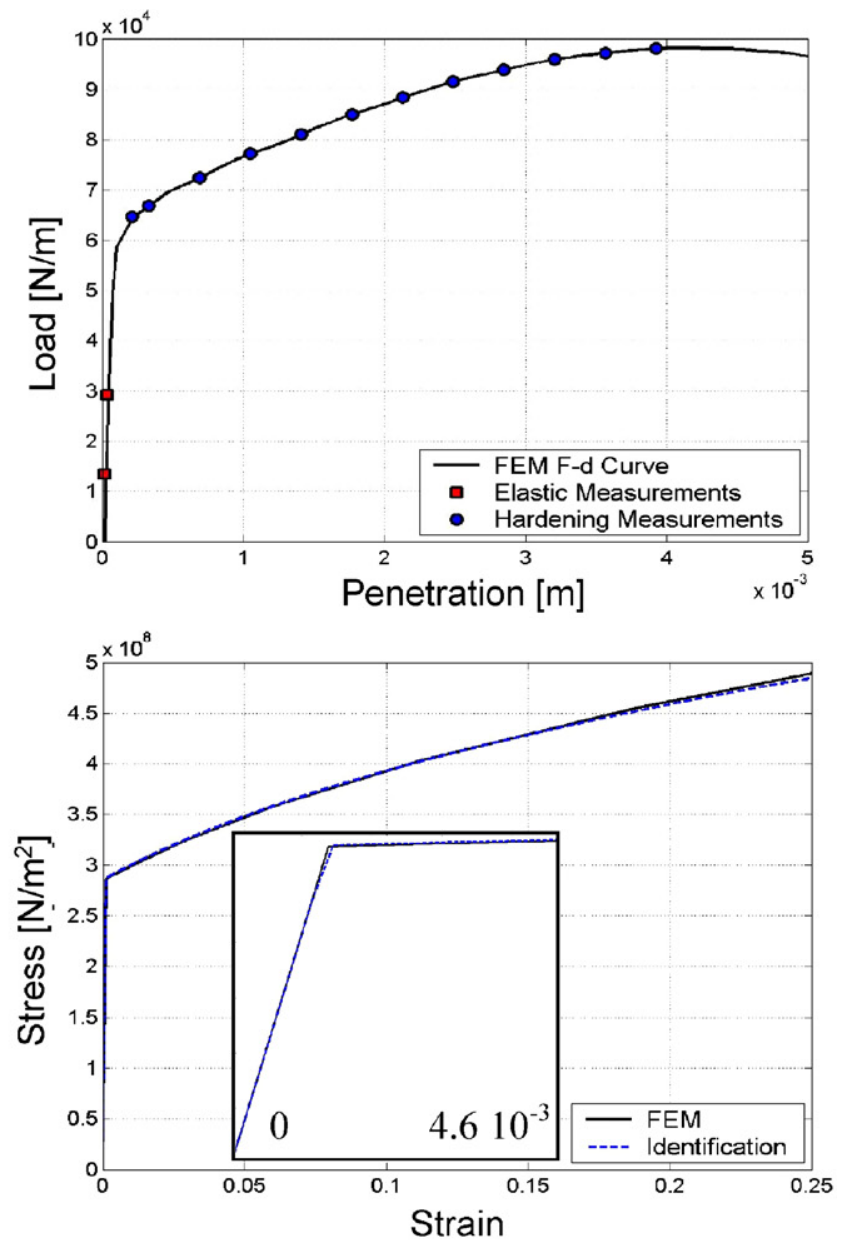

Fig. 8. MATERIAL 1, Swift representation. 
Thanks to the hypothesis of linear deformation across the sheet and the choice of the hardening law (10) which does not include additional constant terms, it is possible to compare directly (12) and (1), and to extract consequently the material hardening parameters.

$$
\left\{\begin{array}{l}
C=\frac{2 \cdot \bar{C}}{\bar{e}+2} \cdot\left(\frac{t}{2}\right)^{\bar{e}+2} \Rightarrow \bar{C}=\frac{C \cdot(\bar{e}+2)}{2 \cdot(t / 2)^{\bar{e}+2}} \\
e=\bar{e}
\end{array}\right.
$$

In conclusion, for each identified bending moment-curvature law constituting a portion of the complete one the parameters of the simplified hardening law (10) are available by relations (13). Then, by means of a method similar to the one described in Fig. 3 for bending moment-curvature identification, it is possible to identify the stress-strain hardening behaviour as a concatenation of curve portions. In other words, using relations (13) for all the identified bending moment-curvature curves (one for each couple of $F-d$ acquisitions) the same number of stress-strain curves are available. Since the said curves are linked each one to different bending levels, it is necessary to determinate the validity field for each curve. This task is resolved similarly to the bending moment-curvature case (Fig. 3) using reference deformations instead than reference curvatures. The reference deformations are generated directly from the reference curvatures employing the usual linear deformation hypothesis:

$\varepsilon_{r, i}=\chi_{r, i} \cdot \frac{t}{2} \cdot f=\varepsilon_{\max , i} \cdot f ; \quad 1 / 3 \leq f \leq 2 / 3$

Since for each reference curvature are available all the deformation values included between 0 and the maximum value $\varepsilon_{\max , i}$, should be supplied a rule to tune the more appropriate reference deformation. The preferred way is reducing the maximum deformation to values near the middle deformation $(1 / 2) \cdot \varepsilon_{\max , i}$, and this is achieved by means of the coefficient $f$ defined in (14). Experience showed that all the values for $f$ provided in (14) furnish satisfactory results for the discussed curve portions composition.

Particularizing the Eq. (13) for the elastic range $(e=\bar{e}=1$, $\bar{C} \Rightarrow E$ ), the Young's modulus $E$, is immediately available.

The last step to perform for the identification of stress-strain is a smoothing procedure applied on all partial curves obtained, and the consequent calculation of the unique Swift's relation:

$\sigma=\bar{C}\left(\varepsilon_{0}+\varepsilon_{\mathrm{p}}\right)^{\bar{e}}$

In principle, the three free parameters $\bar{C}, \varepsilon_{0}, \bar{e}$ in (15) could be determined by means of a non-linear equations system; the equations of this system impose to the relation (15) to include
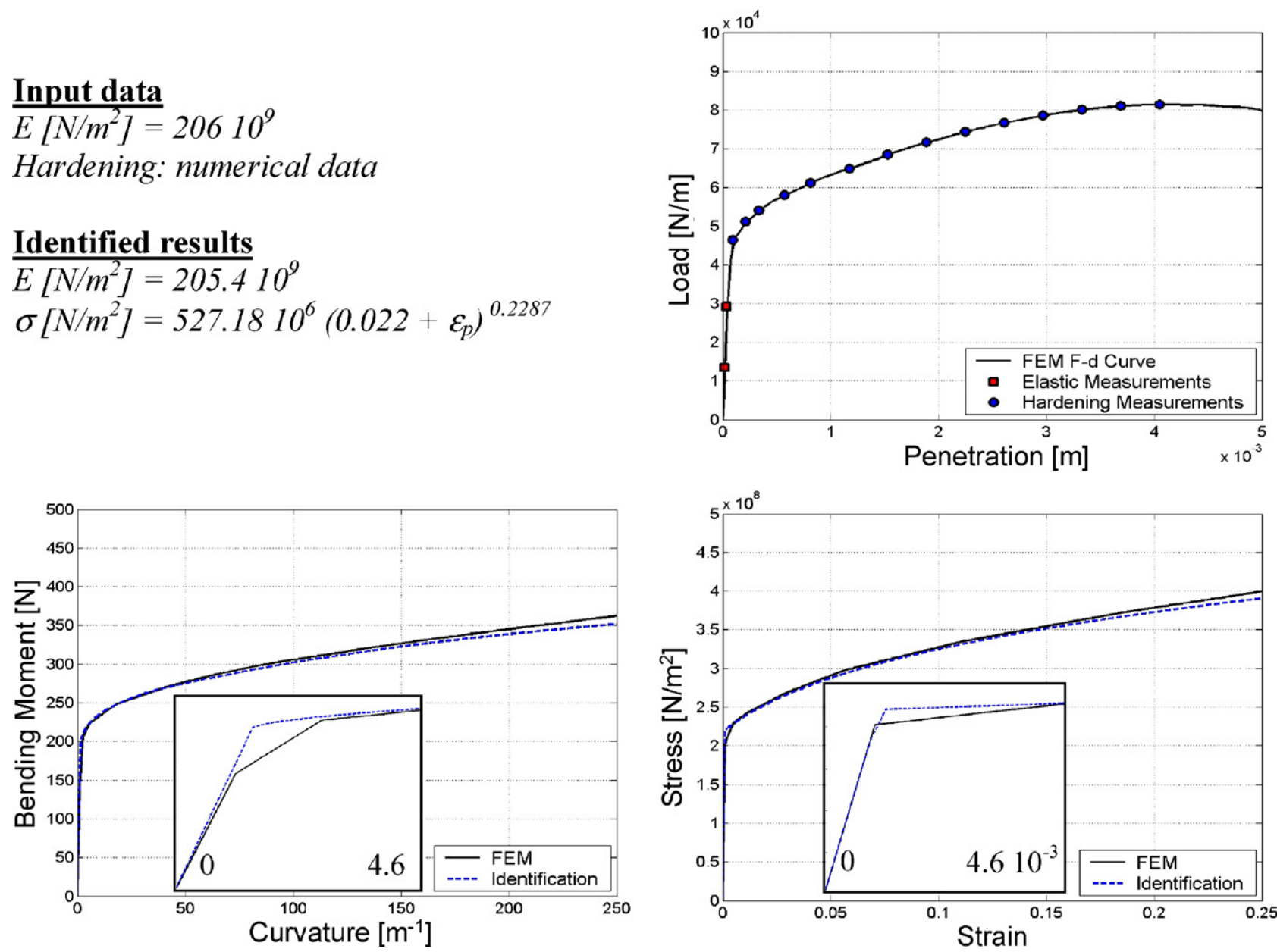

Input data

$E\left[\mathrm{~N} / \mathrm{m}^{2}\right]=20610^{9}$

Hardening: numerical data

\section{Identified results}

$$
\begin{aligned}
E\left[N / m^{2}\right] & =205.410^{9} \\
\sigma\left[N / m^{2}\right] & =527.1810^{6}\left(0.022+\varepsilon_{p}\right)^{0.2287}
\end{aligned}
$$

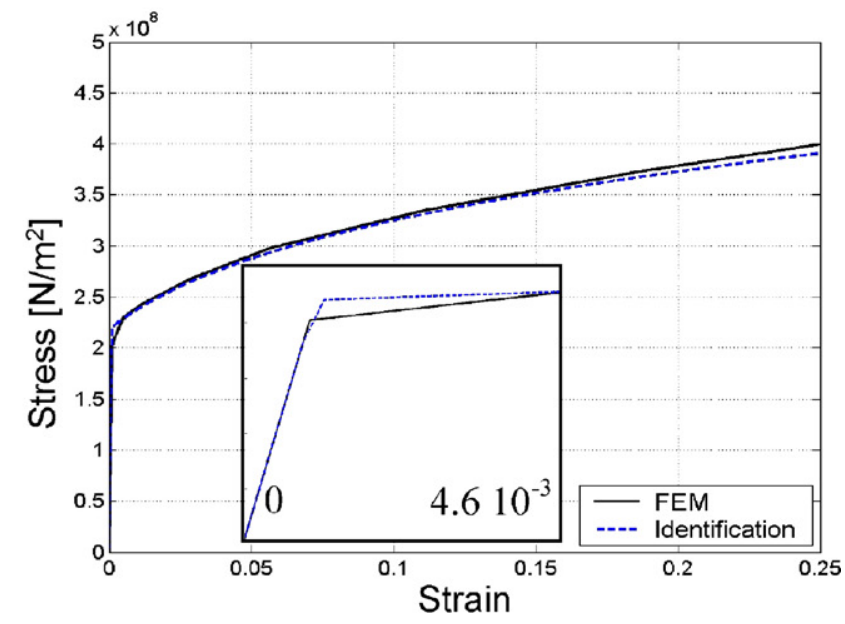

Fig. 9. MATERIAL 2, tensile test. 
three spaced points of the composed $\sigma-\varepsilon$ curve. Practically the point are more than three, in particular if there are $h$ groups of three points, the parameters are calculated as mean of the relative $h$ values.

Obviously, this operation is gainful if the identified material behaviour matches well the three-parametric law (15), but, like mentioned before, this is generally verified for the most part of metal materials.

\section{Numerical comparison}

In order to validate the method, some FEM simulations are executed while respecting the actual operative conditions. At this stage of the work a FEM validation is considered appropriate, as a matter of fact it offers the possibility to test the method limits employing a wide variety of material models, in the absence of any measurement errors; this allows to focus attention only on mathematical modelling validation.

Fig. 5 synthetically shows how FEM simulations are performed to achieve comparisons.

\subsection{FE model}

Taking advantage of geometric symmetric and of inefficacy of $z$ direction (Fig. 4), FEM simulations are executed by means of a 2-D model (Fig. 6) which, adequately loaded and constrained, models the right side of air-bending process. The elements used (PLANE42 in the ANSYS ${ }^{\circledR}$ code) present four nodes with 2 d.o.f.'s (displacements on element lying plane), and Coulomb friction is used for the sheet-die contact analysis. The model is loaded by means of increasing penetration ( $d$ in Fig. 1b), effective load applied results in an increasing-decreasing path. During the whole process the force-penetration data are stored and the obtained curve is consequently elaborated as would occur for experimental reference data.

It is interesting to investigate on the reliability of linear deformation hypothesis across the sheet thickness. To this goal a FEM simulation is executed in order to analyse the deformation variation across the thickness just below the punch when increasing the curvature $\chi$ : results are shown in Fig. 7.

Fig. 7 reveals that deformation increases towards curvature, and linearity assumption becomes more and more inappropriate, but it also shows that, in spite of the small thickness of sheets, the hypothesis carried on is almost always realistic. In consideration of these results, the linearity assumption is considered appropriate within the deformation limits provided by Fig. 7c, where maximum deformation experienced is approximately 0.22 . It is therefore substantially the same limit previously imposed to stress-strain identification by relation (11) $\left(\varepsilon=0.25 \rightarrow \varepsilon_{\log } \approx 0.22\right)$.
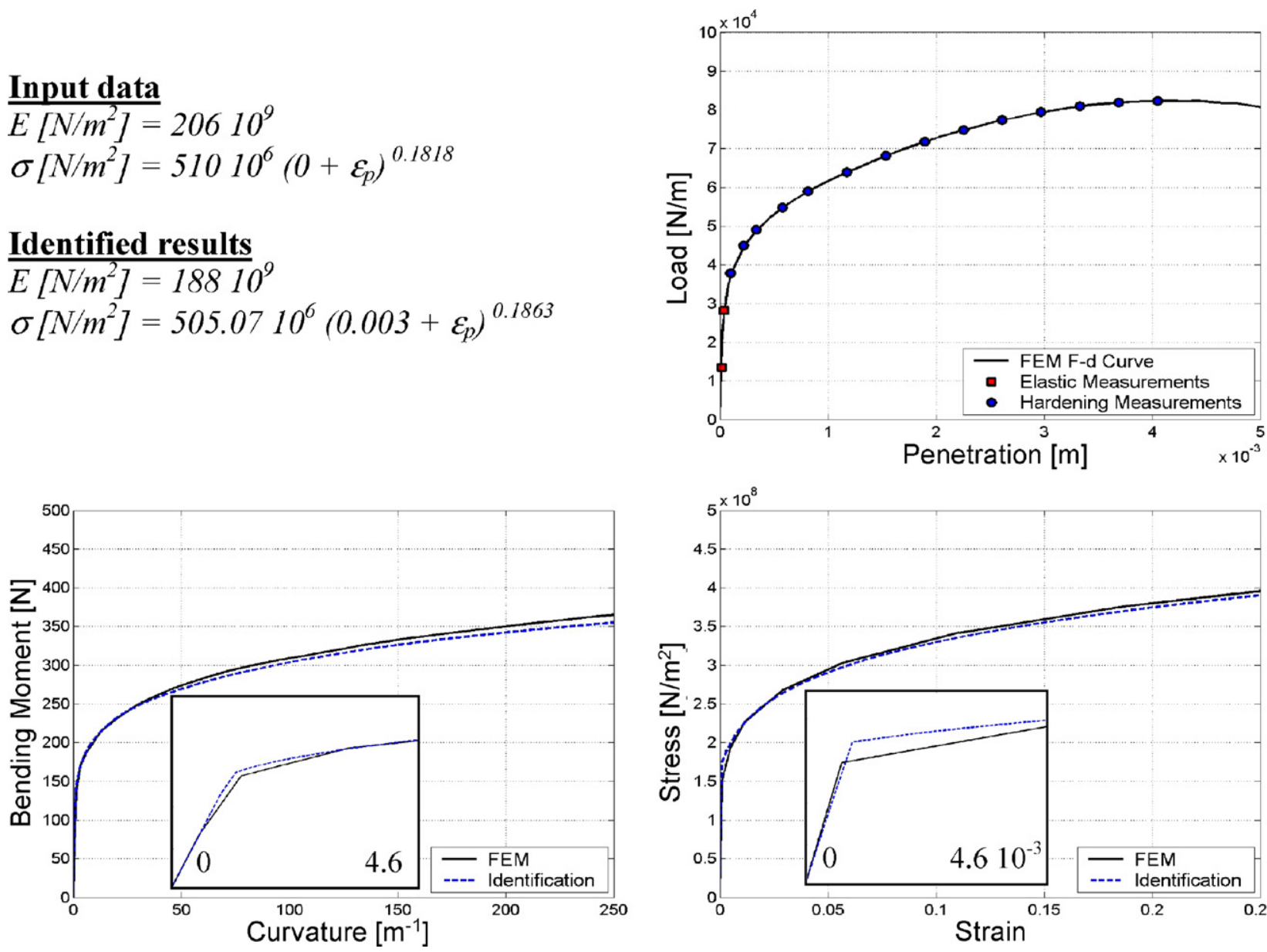

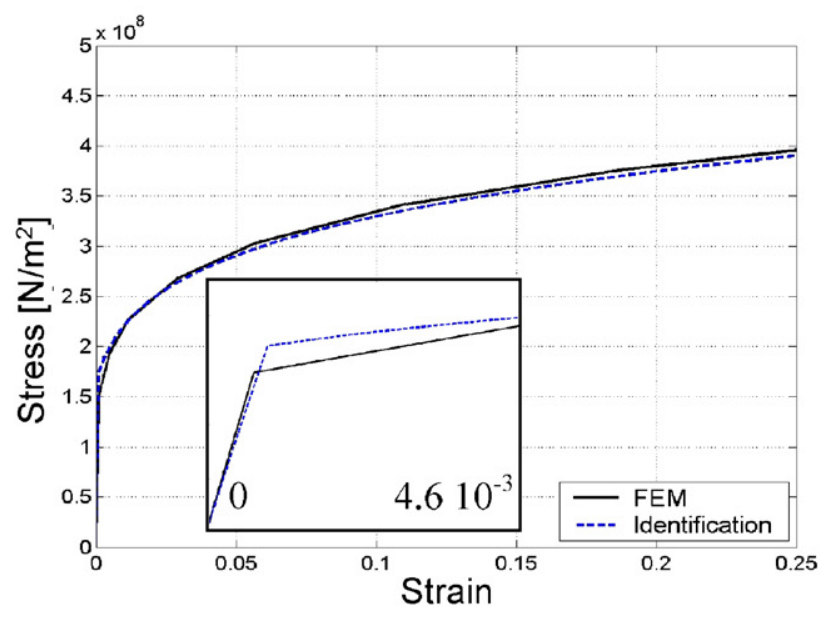

Fig. 10. MATERIAL 3, pure exponential law. 


\section{Input data}

$E\left[\mathrm{~N} / \mathrm{m}^{2}\right]=20610^{9}$

$\varepsilon_{1}=1.3310^{-3}$

$\sigma_{1}\left[\mathrm{~N} / \mathrm{m}^{2}\right]=27510^{6}$

$m_{1}\left[N / m^{2}\right]=465.3410^{6}$

\section{Identified results}
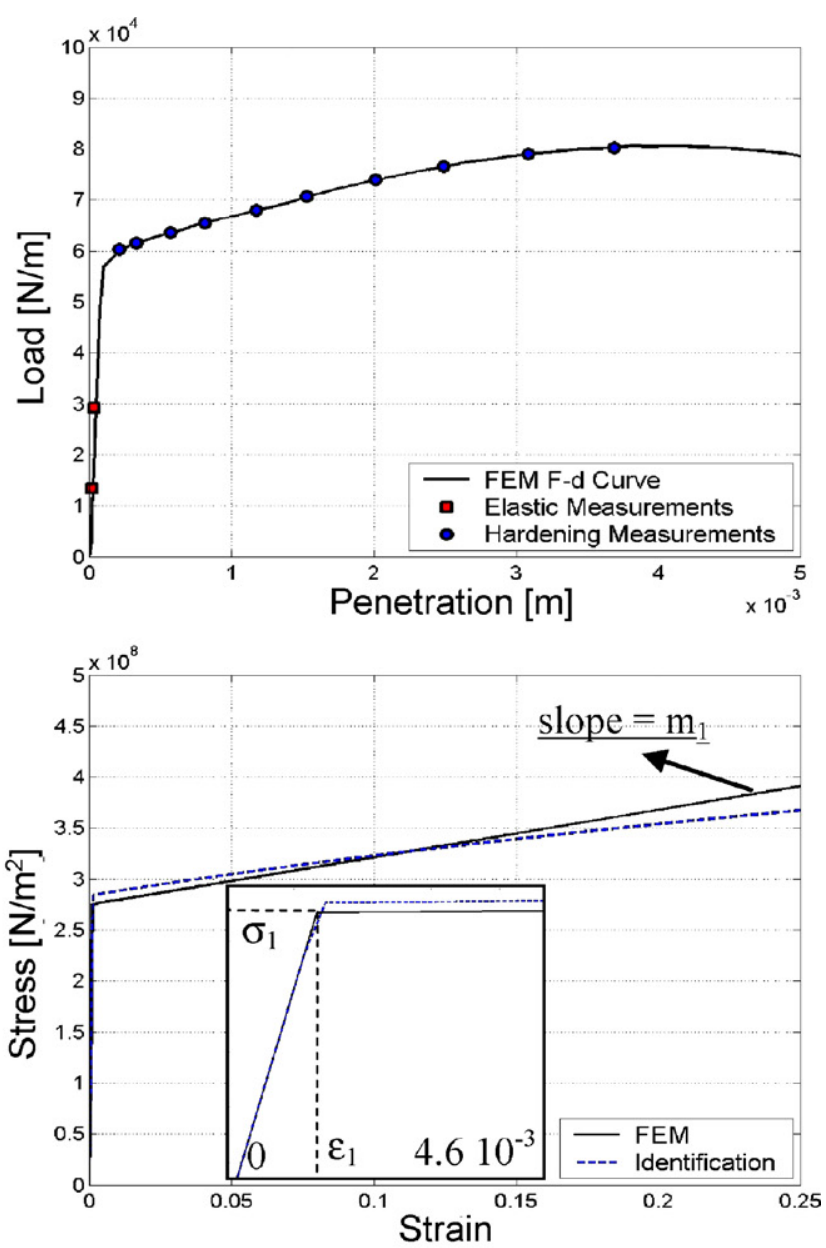

Fig. 11. MATERIAL 4, bilinear.

\subsection{Results and discussion}

FEM simulations are executed using different material types with the aim to test algorithm flexibility in material identification, and its aptitude to identify bending moment-curvature behaviour by means of a composition of purely exponential curve portions.

All the comparisons hereinafter are presented by pictures containing input and identified values, punch load versus penetration, bending moment towards curvature, true stress-true strain curve.

The material 1 (Fig. 8) taken from [8], is a typical metalforming steels. It presents an hardening behaviour which follows the Swift's law, and since the final stress-strain result is expressed by the same law (15), for this steel a numeric comparison of the Swift's parameters is also possible.

Instead, steel 2 (Fig. 9) is presented in numeric form directly from an experimental uni-axial tensile test. This hardening behaviour appears lightly different from the previous ones, mainly for a smoother shape.

The material 3 (Fig. 10) is governed by a purely exponential law, like in Eq. (10), and the same operating method is used here; first the bending moment-curvature behaviour is determined by concatenation of purely exponential curve portions (1) and then, as usual, the same treatment is reserved for the stress-strain curve which is at the end fitted by Swift's law (15). It is shown as in this case the parameter $\varepsilon_{0}$ of the mentioned law, as it would be expected, assumes a value very close to 0 .

Material model 4 (Fig. 11) follows a bilinear law, and although employed in simplified models [1], it represents adequately the material behaviour only in few cases, it is however

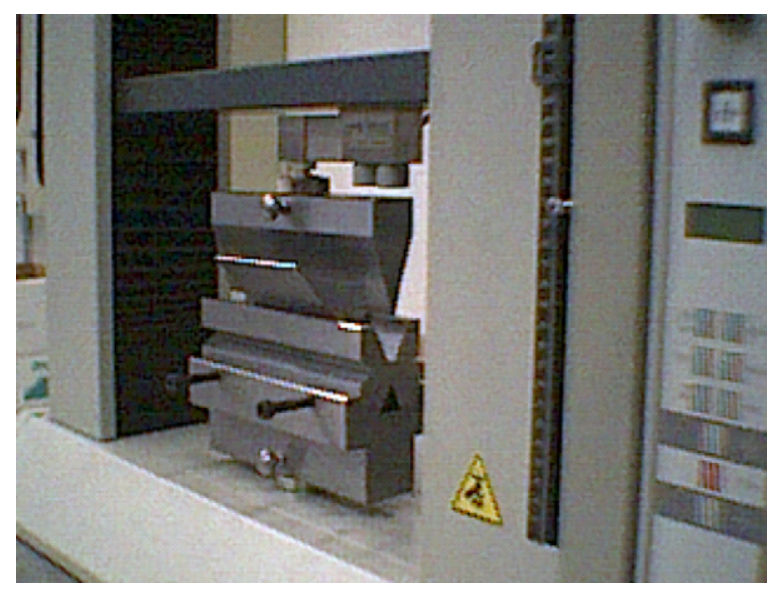

Fig. 12. Experimental apparatus. 


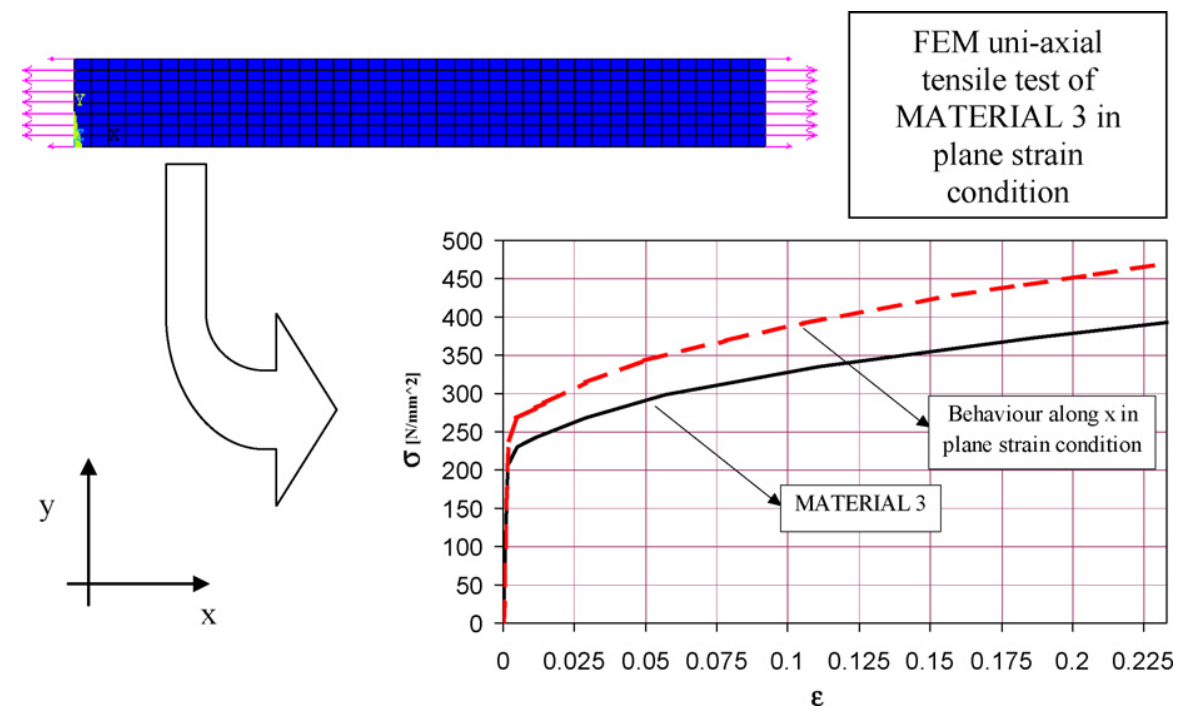

Fig. 13. Material behaviour in plane strain condition.

used because it involves several analytic simplifications. In this particular case here discussed, it is considered to highlight identification method limits, since it is not easy to adapt exponential curves to straight lines.

As explained above, force-penetration are the main input data for the algorithm proposed which, taking input from some val-

\section{Material data}

$E_{p s}=E /\left(1-v^{2}\right)=226.310^{9} \mathrm{~N} / \mathrm{m}^{2}$

Hardening: numerical data

\section{Identified results}

$$
\begin{aligned}
& \frac{\text { Ident }}{E_{p s}\left[\mathrm{~N} / \mathrm{m}^{2}\right]=216.310^{9}} \\
& \sigma\left[\mathrm{N} / \mathrm{m}^{2}\right]=614.2610^{6}\left(0.006+\varepsilon_{p}\right)^{0.194}
\end{aligned}
$$

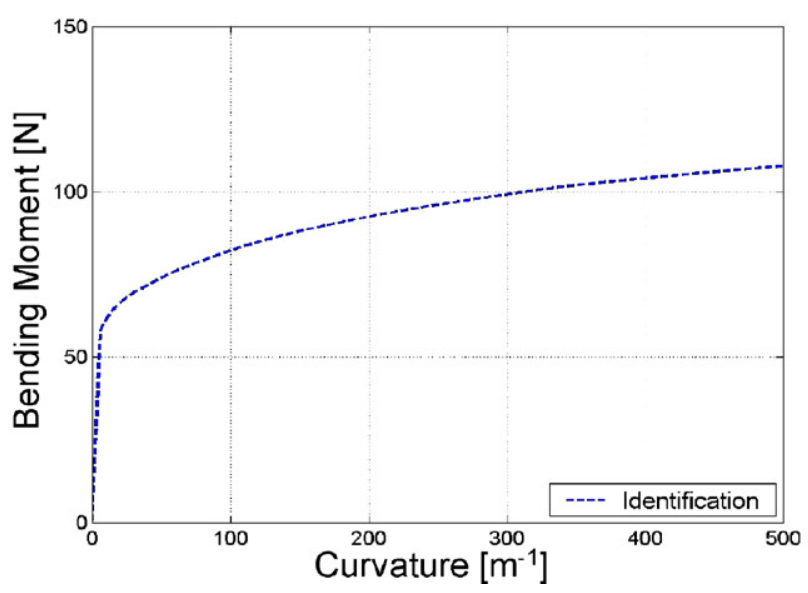

ues in the elastic zone and some values in the hardening one, and using the air-bending geometry and the predetermined friction coefficient, executes the computation. It is interesting to note that force-penetration behaviour is similar to the stress-strain one, and this involves the possibility to choose appropriately the zone where it is favourable to increase measurements acquisi-
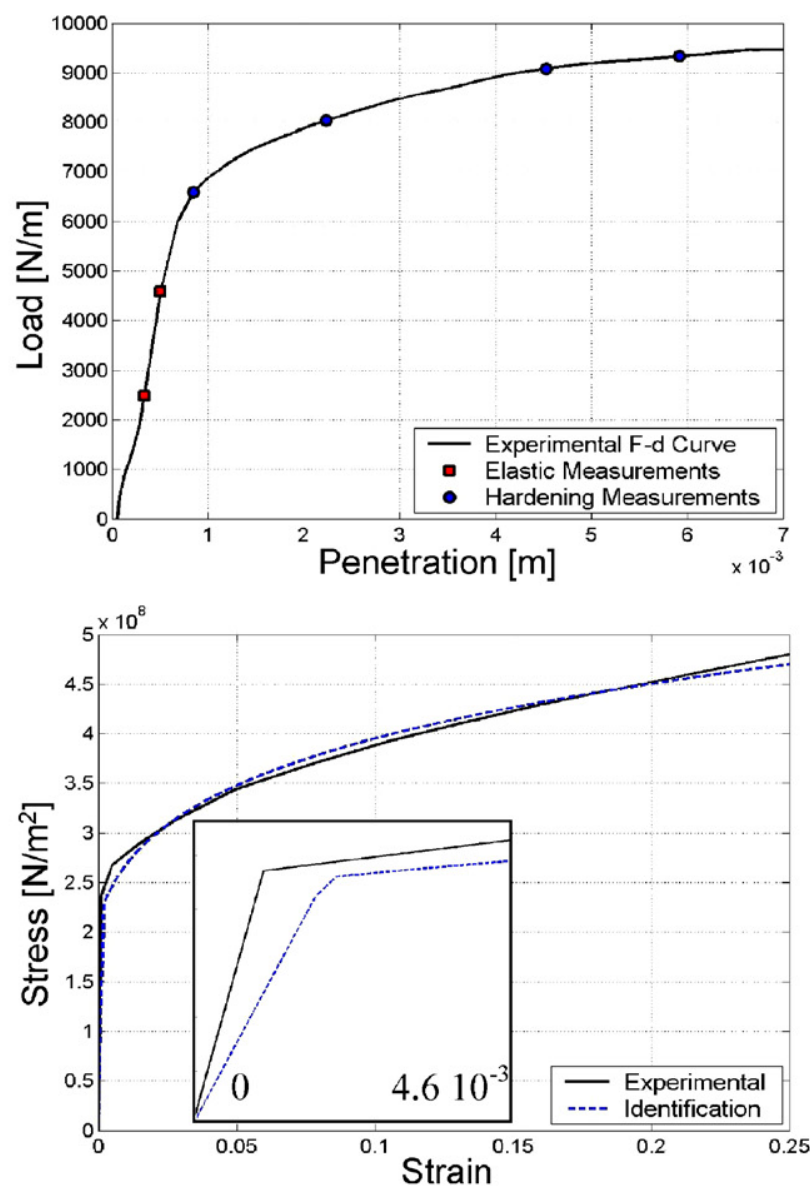

Fig. 14. Material identification by an experimental test (material 2 in plane strain condition). 
tion. In facts, where force-penetration curve presents a strong change of slope it is appropriate to increase the measurement data since this will advantage the identification smoothing on the corresponding stress-strain region. Usually 2 measurements for the elastic zone, and 12-14 measurements for the hardening zone are enough to obtain accurate material identification. These points, as Figs. 8-11 show, are taken within the limits imposed by the linearity deformation hypothesis discussed above.

The numerical results, the graphic comparisons of bending moment-curvature and stress-strain and the related zooms on the elastic zone limit, demonstrate a general suitable algorithm behaviour, especially for materials 1-3 (Figs. 8-10). Instead the model finds some limits for materials 4 (Fig. 11), where the chosen bilinear behaviour is very hard to fit by exponential laws.

\section{Experimental test}

In order to show the method application in a real case, in this section are synthetically illustrated the identification results carried out on a actual airbending test. The test is executed by means of a standard tensile test machine, equipped with typical sheet bending tools, as shown in Fig. 12.

The sheet material behaviour is known, since it is the material 2 tested by the uni-axial tensile test and used in the FEM models showed in Fig. 9. This makes possible, after some modelling, to compare the resulting stress-strain curves.

Since the model is able to identify the material behaviour along $x$ direction (Fig. 4) independently from the plane-stress/strain condition, and given that the geometric dimensions of the specimen suggest that experimental bending is executed under plane strain condition $(L=80 \mathrm{~mm}, t=1 \mathrm{~mm})$ it is possible to conclude that the identified material in this case is the stress-strain behaviour in plane strain condition. Consecutively, the final curves comparison should be made between the identified one and the stress-strain behaviour of tested material in plane strain condition.

Using FEM it is possible to re-perform an uni-axial tensile test now under plane strain condition. The resulting true stress-true strain curve (Fig. 13), effectively constitutes the curve to compare to the one identified during the process.

It is important to highlight that, since the number of measurements processed $(m=4)$ is limited and the consequent computation is quick, the identification can be conducted in a real time execution, supplying however very satisfactory comparison results (Fig. 14).

\section{Conclusions}

A new identification method of material elasto-plastic characteristics, based on sheet air-bending, is proposed and tested. Method input data are the actual force-penetration values experienced during the bending, geometric features, and the sheet-die friction coefficient. By means of a multi-joint bending modelling and a simplified exponential law describing the joints hardening behaviour, the bending moment-curvature law is identified in numerical form. Then, in a first step through numerical form and then using Swift's analytical law, the true-stress versus truestrain relation is calculated benefiting from the hypothesises of linear deformation across the sheet and plane-stress condition. Although in principle only 2 force-penetration measurements are required for the hardening identification, the problem is solved using more measurements (12-14) sampled inside the whole penetration phase, and the consequential hardening law is the composition of six to seven curve portions resulting from the elaboration of couples of force-penetration points. The valida- tion tests performed regards FEM simulation and experiments. The FEM ones are performed to test the limits of the mathematical method. The experimental test is carried out to demonstrate the procedure feasibility, and the possibility to manage both plane strain and plane-stress conditions.

Generally the method demonstrates a suitable behaviour for both test typologies, as results show. The composition technique which considers portions of purely exponential curve is able to adapt to real and unpredictable hardening behaviour, both in terms of bending moment-curvature and stress-strain.

Eventual smoothing on data to get an unique Swift's law provides a more manageable stress-strain curve making it possible further predictable simulations of air-bending processes.

If only springback should be controlled, the multi-joint model allows to manage also bimetallic sheets or materials presenting an influential surface covering, the worthiness of the identification is then limited to bending moment-curvature data but on-line bending control can be performed.

Finally, as experimental tests show, the method is also usable on a very real time basis, taking account of few measurement points and thus profiting of the reduced computation time; therefore, springback compensation of the sheet under bending is possible using the material identified from time to time.

\section{References}

[1] P.S. Raghupathi, M. Karima, N. Akgerman, T. Altan, A simplified approach to calculate springback in brake bending, in: 11th NAMRC, Wisconsin, 1983.

[2] Z. Tan, B. Persson, C. Magnusson, Plastic bending of anisotropic sheet metal, Int. J. Mech. Sci. 37 (1995) 405-421.

[3] C. Wang, G. Kinzel, T. Altan, Mathematical modeling of plane strain bending of sheet and plate, J. Mater. Process. Technol. 39 (1993) 279304.

[4] W.B. Lee, K.C. Chan, J. Chakrabarty, An exact solution for the elastic/plastic bending of anisotropic sheet metal under condition of plane strain, Int. J. Mech. Sci. 43 (2001) 1871-1880.

[5] L.J. De Vin, A.H. Streppel, U.P. Singh, H.J.J. Kals, A process model for air bending, J. Mater. Process. Technol. 57 (1996) 48-54.

[6] W.B. Lee, K.C. Chan, J. Chakrabarty, An analysis of the plane-strain bending of an orthotropic sheet in the elastic/plastic range, J. Mater. Process. Technol. 104 (2000) 48-52.

[7] M.V. Inamdar, P.P. Date, U.B. Desai, Studies on the prediction of springback in air vee bending of metallic sheets using an artificial neural network, $\mathrm{J}$. Mater. Process. Technol. 108 (2000) 45-54.

[8] You-Min Huang, Daw-Kwei Leu, Effects of process variables on V-die bending process of steel sheet, Int. J. Mech. Sci. 40 (1998) 631-650

[9] V.V. Toropov, F. Yoshida, E. van der Giessen, Material parameter identification for large plasticity models, in: EUROMECH 357, Kerkrade, 1997.

[10] F. Yoshida, M. Urabe, V.V. Toropov, Identification of material parameters in constitutive model for sheet metals from cyclic bending tests, Int. J. Mech. Sci. 40 (1998) 237-249.

[11] F. Yoshida, M. Urabe, R. Hinoa, V.V. Toropov, Inverse approach to identification of material parameters of cyclic elasto-plasticity for component layers of a bimetallic sheet, Int. J. Plast. 19 (2003) 2149-2170.

[12] K.M. Zhao, J.K. Lee, Generation of cyclic stress-strain curves for sheet metals, J. Eng. Mater. Technol. 123 (2001) 391-397.

[13] K.A. Stelson, Real time identification of workpiece-material characteristics from measurements during brake forming, Trans. ASME J. Eng. Ind. 105 (1983) 45-53.

[14] K.A. Stelson, An adaptive pressbrake control for strain hardening materials, Trans. ASME, J. Eng. Ind. 108 (1986) 127-132. 
[15] L. Geng, Y. Shen, R.H. Wagoner, Anisotropic hardening equations derived from reverse-bending testing, Int. J. Plast. 18 (2002) 743-767.

[16] K. Anokye-Siribor, U.P. Singh, A new analytical model for pressbrake forming using in-process identification of material characteristics, J. Mater. Process. Technol. 99 (2000) 103-112.
[17] A. Chandra, Real-time identification and control of springback in sheet metal forming, Trans. ASME, J. Eng. Ind. 109 (1987) 265-273.

[18] L. Antonelli, P. Salvini, F. Vivio, V. Vullo, A new tested method for adaptive control of springback in air bending, in: 12th ICEM, Bari, 2004. 